\title{
5. Engagiert bis ins hohe Alter? Organisationsgebundenes ehrenamtliches Engagement in der zweiten Lebenshälfte
}

\author{
Martin Wetzel \& Julia Simonson
}

\section{Kernaussagen}

Der Anteil von Personen, die ein ehrenamtliches Engagement ausüben, hat sich unter den 40- bis 85-Jährigen zwischen den Jahren 1996 und 2014 verdoppelt: Während im Jahr 1996 etwa jede zehnte Person (11,7 Prozent) im Rahmen einer Organisation ehrenamtlich engagiert war, ist es im Jahr 2014 schon fast jede vierte (22,2 Prozent). Dieser Zuwachs ist unter Personen im höheren Lebensalter besonders deutlich.

Im Jahr 2014 zeigen sich deutliche Unterschiede im ehrenamtlichen Engagement nach Geschlecht, Bildung, Landesteil und Erwerbsstatus: Männer sind 1,3 Mal häufiger engagiert als Frauen. Dieser Geschlechtsunterschied war 1996 noch größer. Hochgebildete sind im Jahr 2014 3,6 Mal häufiger ehrenamtlich engagiert als Niedriggebildete, was sich seit 1996 nicht verändert hat. Westdeutsche engagieren sich 1,7 Mal häufiger ehrenamtlich als Ostdeutsche. Der Unterschied zwischen den Landesteilen ist seit 1996 stabil. Erwerbstätige und Personen im Ruhestand engagieren sich häufiger als Nicht-Erwerbstätige.

Im Jahr 2014 ist jede vierte ehrenamtlich engagierte Person im Alter von $\mathbf{5 0}$ bis $\mathbf{8 5}$ Jahren in einer Seniorengruppe aktiv: Jüngere engagieren sich seltener in seniorenspezifischen Gruppen als Ältere. Zwischen den Jahren 1996 und 2014 ist der Anteil von Engagierten in Seniorengruppen an der Bevölkerung von 2,1 Prozent auf 5,6 Prozent gestiegen. Das Verhältnis zwischen seniorenspezifischem und allgemeinem Engagement hat sich dabei jedoch nicht verändert.

Ein Drittel der im Jahr 2014 ehrenamtlich Engagierten hat in den letzten sechs Jahren ein neues Engagement aufgenommen und zwei Drittel üben ihr Engagement schon länger als sechs Jahre aus: Im Jahr 2014 haben 7,1 Prozent der Bevölkerung im Alter von 40 bis 85 Jahren ein Engagement ausgeübt, welches sie frühestens 2009 begonnen hatten. Ältere nehmen zu einem geringeren Anteil ein Engagement neu auf als Jüngere. Zudem üben Ältere ihr Engagement zu einem größeren Teil bereits über einen längeren Zeitraum aus als Jüngere. Zwischen 1996 und 2014 hat sich der Anteil der neu Engagierten an allen Engagierten nicht bedeutsam verändert.

\subsection{Einleitung}

Freiwilliges und ehrenamtliches Engagement kann zum Zusammenhalt der Gesellschaft beitragen und sozialintegrativ wirken (Zimmer \& Rauschenbach 2011). Personen in der zweiten Lebenshälfte können über ehrenamtliches En- gagement ihre Erfahrungen und Kompetenzen einbringen und somit einen positiven Beitrag für die Gesellschaft leisten. Das freiwillige und ehrenamtliche Engagement von Älteren wird daher sowohl in Deutschland (zum Beispiel Fünf- 
ter Altenbericht 2006), als auch auf europäischer Ebene (zum Beispiel EU 2012) sowie im internationalen Kontext (zum Beispiel World Health Organization 2002) als gesellschaftliches Potenzial thematisiert. Personen, die sich ehrenamtlich engagieren, können davon aber auch persönlich profitieren. Verschiedene Studien haben gezeigt, dass Engagement zu mehr individuellem Wohlbefinden beiträgt (für Gesundheit: Everard, Lach et al. 2000; für Lebenszufriedenheit: van Willigen 2000). Darüber hinaus ist ehrenamtliches Engagement eine zentrale Form gesellschaftlicher Teilhabe. Allerdings kann sich ehrenamtliches Engagement in einigen Fällen auch nachteilig auf Wohlbefinden und Gesundheit auswirken, nämlich dann, wenn damit Stress und Überforderung einhergehen (Windsor, Anstey et al. 2008), aber auch, wenn damit eine gesellschaftliche Norm zum ,aktiven Altern' verbunden wird, an der nicht alle Menschen gleichermaßen teilhaben können (van Dyk 2009).

Ehrenamtliches Engagement kann also Auswirkungen auf die Lebenssituation und auf das Wohlbefinden der engagierten Personen, auf die Situation der Zielgruppen des Engagements sowie auf die Gesellschaft insgesamt haben. Engagement ist aber auch an Voraussetzungen geknüpft (Simonson, Hagen et al. 2013). Gesellschaftliche Rahmenbedingungen und individuelle Merkmale haben einen Einfluss auf Ressourcen und Gelegenheitsstrukturen, welche ehrenamtliches Engagement begünstigen oder hemmen (vgl. Gesthuizen \& Scheepers 2012). So besitzen Personen mit einem höheren Bildungsniveau auch mehr individuelle Ressourcen als Personen mit einem niedrigeren Bildungsniveau, wie zum Beispiel mehr Einkommen, höhere Lebenszufriedenheit, bessere Gesundheit und höhere Selbstwirksamkeit (Ross \& Wu 1995; Laubach, Schumacher et al. 2000) sowie vorteilhaftere soziale Netzwerke, welche sich wiederum positiv auf die Wahrscheinlichkeit auswirken, ein ehrenamtliches Engagement auszuüben (zum Beispiel Wilson \& Musick 1997; Wilson 2000; Hank \& Stuck 2008; Alscher, Dathe et al. 2009; Lancee \& Radl 2014). Ebenfalls können unterschiedliche Gelegenheitsstrukturen relevant sein. So üben beispielsweise Erwerbstätige häufiger ein ehrenamtliches Engagement aus als Arbeits- lose (Alscher, Dathe et al. 2009; Gesthuizen \& Scheepers 2012). Wenn ehrenamtliches Engagement sich positiv auf die Lebensqualität von Personen auswirkt, dann führt ein ungleicher $\mathrm{Zu}$ gang zu ehrenamtlichem Engagement folglich $\mathrm{zu}$ ungleichen Lebenschancen. Die genannten Mechanismen können somit soziale Ungleichheiten verstärken. Dieses Kapitel widmet sich der Frage, inwieweit das ehrenamtliche Engagement Älterer in den letzten Jahren zugenommen hat, wer in der zweiten Lebenshälfte einem ehrenamtlichen Engagement nachgeht und welche Gruppen besonders starke Veränderungen aufweisen.

Empirische Studien zeigen deutliche Unterschiede zwischen den Engagementquoten unterschiedlicher Gruppen. Beispielsweise engagieren sich Personen in Ostdeutschland seltener als in Westdeutschland (Naumann \& Romeu Gordo 2010), was meist mit unterschiedlichen Gelegenheitsstrukturen begründet wird. So bestehen beispielsweise noch deutliche Unterschiede in der Erwerbsstruktur zwischen den beiden Landesteilen (vgl. Kapitel 3). Unterschiede im ehrenamtlichen Engagement scheinen auch zwischen den Geschlechtern zu bestehen. Dass Frauen sich seltener als Männer (Alscher, Dathe et al. 2009) engagieren, könnte daran liegen, dass Frauen durch ihre geringere Arbeitsmarktintegration seltener in Kontakt mit Möglichkeiten für ehrenamtliches Engagement kommen (von Rosenbladt 2000; Naumann \& Romeu Gordo 2010; Lancee \& Radl 2014; Quaranta 2015). Darüber hinaus führt die immer noch verbreitete geschlechtsspezifische innerfamiliäre Arbeitsteilung dazu, dass Frauen durch Haushalts- und Sorgetätigkeiten, zusätzlich zur Erwerbstätigkeit, häufig unter einer Doppelbelastung stehen (vgl. Kapitel 12 und 13), die ihnen weniger Zeit für ehrenamtliches Engagement lässt als Männern.

Trotz der positiven Befunde hinsichtlich des Zusammenhangs von Erwerbstätigkeit und Ehrenamt scheint der Übergang in den Ruhestand ehrenamtliches Engagement quantitativ nur wenig (Mutchler, Burr et al. 2003; Erlinghagen 2010) oder gar nicht zu beeinflussen (Scherger, Nazroo et al. 2011; Lancee \& Radl 2014). Selbst wenn sich mit dem Ruhestandsübergang nicht die Engagementquoten verändern, könnte es jedoch sein, dass es 
zu einer inhaltlichen Verschiebung kommt - zum Beispiel von allgemein ausgerichteten $\mathrm{zu}$ seniorenspezifischen Organisationen. Auswertungen des Deutschen Freiwilligensurveys (FWS) haben gezeigt, dass sich ältere Personen eher für Ältere engagieren und jüngere Personen eher für Jüngere (Hagen \& Vogel 2012). Eine strukturelle Verschiebung zum Engagement in seniorenspezifischen Gruppen ist besonders dann interessant, wenn die Teilnahme an seniorenspezifischen Gruppen anderen Mustern unterworfen ist, als dies allgemein beim Ehrenamt der Fall ist, oder sich diese Form des Engagements in ihren Auswirkungen auf die engagierten Personen unterscheidet.

In diesem Kapitel wird das ehrenamtliche Engagement unterschiedlicher gesellschaftlicher Gruppen betrachtet. Im Zentrum stehen dabei Unterschiede zwischen Altersgruppen, Geschlechtern, Bildung und Landesteilen (Ostbeziehungsweise Westdeutschland) sowie der Erwerbsstatus, da mit ihnen spezifische Ressourcen und Gelegenheitsstrukturen einhergehen. Weiterhin werden die Veränderungen zwischen den Jahren 1996 und 2014 betrachtet, sodass allgemeine Trends, aber auch Annäherungs- und Distanzierungstrends zwischen verschiedenen Gruppen untersucht werden können.

Von 1996 bis 2008 kam es bereits zu einer Zunahme von ehrenamtlichem Engagement (vgl. Naumann \& Romeu Gordo 2010). Diese Zunahme könnte erstens mit einem politisch intendierten Wandel zu einer verbesserten ,Engagementkultur ' zusammenhängen. Seit Ende der 1990er Jahre, beginnend mit der Einsetzung einer Enquete-Kommission ,Zukunft des bürgerschaftlichen Engagements' hat sich die Engagementpolitik als eigenständiges Politikfeld herausgebildet (vgl. Hartnuß, Olk et al. 2011). Freiwillige und ehrenamtliche, unentgeltlich ausgeübte Tätigkeiten wurden zunehmend als gesellschaftliche Ressource entdeckt und Engagement sollte durch politische Maßnahmen gefördert werden. Bisher wurden zur Förderung des Engagements beispielsweise im Jahr 2005 der unfallversicherungsrechtliche Schutz Engagierter verbessert sowie im Jahr 2011 der Bundesfreiwilligendienst eingeführt, der allen Altersgruppen offen steht. Weitere Initiativen richteten sich speziell an Ältere, wie beispielsweise das Programm, Aktiv im Alter.
Diese Maßnahmen verändern die Rahmenbedingungen für Engagement und können zu einer zunehmenden Beteiligung von Menschen im Engagement beitragen.

Außerdem hat sich die Arbeitsmarktpolitik zwischen 1996 und 2014 grundsätzlich gewandelt, von einer Periode des frühen Vorruhestands zu einer Zeit der längeren Arbeitsmarktintegration von älteren Erwerbspersonen (vgl. Kapitel 3). Damit steigt der Anteil von Erwerbstätigen unter den älteren Erwerbspersonen und es kommt möglicherweise zu einer Erweiterung der Gelegenheitsstrukturen für Engagement.

Als dritte Entwicklung in der Zeit von 1996 bis 2014 lässt sich feststellen, dass Personen gesundheitsbezogene Ressourcen besser bis ins hohe Alter aufrechterhalten können (vgl. Kapitel 8 und 9). Die Lebensjahre, in denen sich eine Person bei guter Gesundheit befindet, haben sich seit dem Jahr 1996 vor allem nach dem Ruhestandsübergang deutlich erhöht. Diese Gesundheitsressourcen können dazu beitragen, dass gerade Personen im hohen Alter häufiger noch ein Engagement ausüben können. Schließlich haben sich in den letzten Jahren die Sichtweisen auf das eigene Älterwerden verändert (vgl. Kapitel 22). Mit dem höheren Lebensalter sind heute durchaus auch Erwartungen an künftige Entwicklungsmöglichkeiten verknüpft. Freiwilliges und ehrenamtliches Engagement gilt zunehmend als Handlungsoption auch im höheren Alter.

Im Folgenden wird zunächst betrachtet, wie sich die Quoten ehrenamtlichen Engagements in verschiedenen Altersgruppen von 1996 bis 2014 verändert haben. Dabei wird untersucht, ob sich nach Bevölkerungsgruppen (Geschlecht, Bildung, Landesteil, Erwerbsstatus) unterschiedliche Trends zeigen und ob einige Gruppen stärkere Veränderungen aufweisen als andere. In einem zweiten Schritt wird untersucht, ob sich in seniorenspezifischen Organisationen andere Personengruppen ehrenamtlich engagieren als in allgemeinen Organisationen. Es ist zu vermuten, dass sich Personen im höheren Alter häufiger auch in seniorenspezifischen Gruppen engagieren. Jedoch ist derzeit noch unklar, ob sich diese Gruppen in ihrer Zusammensetzung nach Bildung, Geschlecht und Landesteil vom allgemeinen Engagement unterscheiden. Schließlich 
wird der Engagementbeginn betrachtet. Damit steht im Zentrum, ob eine Person in den letzten sechs Kalenderjahren ein ehrenamtliches Engagement aufgenommen hat. Dadurch können Rückschlüsse gezogen werden, in welchen Lebensphasen Personen ein Engagement aufnehmen, ob sich die Zeiten des Neubeginns von Engagement über die Jahre gewandelt haben und ob sich der Anteil der eher neuen gegenüber den eher dauerhaft Engagierten verändert hat. Diese Unterscheidung hilft dabei mögliche Veränderungen der Engagementquote von 1996 bis 2014 besser zu verstehen. Sie verdeutlicht, ob eine Veränderung in der Engagementquote stärker damit zu begründen ist, dass Personen häufiger beziehungsweise seltener ein ehrenamtliches Engagement aus einer früheren Lebensphase fortsetzen. Sie zeigt aber auch auf, ob Personengruppen wie beispielsweise ältere Personen häufiger beziehungsweise seltener ein neues Engagement beginnen.

\subsection{Datengrundlage ${ }^{1}$}

Daten. Ehrenamtliches Engagement wurde im mündlichen Interview (CAPI) des Deutschen Alterssurveys (DEAS) in den Jahren 1996, 2002, 2008 und 2014 erhoben. Da nicht in allen Erhebungsjahren Informationen zu Engagement außerhalb von Organisationen erhoben wurde, beschränken sich die Analysen in diesem Kapitel auf das organisationsgebundene Engagement. Das heißt, dass sonstige Engagementfelder, wie beispielsweise eine Schöffentätigkeit oder Elternvertretung, nicht betrachtet werden. Da in den letzten Jahrzehnten besonders nicht-organisationsgebundenes Engagement (beispielsweise in Initiativen oder Projekten) häufiger geworden ist (Sachße 2011), verfolgt dieses Kapitel also eher einen konservativen Ansatz bei der Betrachtung der aktuel-

1 Die Daten des DEAS können für wissenschaftliche Zwecke kostenlos beim Forschungsdatenzentrum des DZA (www.fdz-dza.de) bezogen werden.
Im vorliegenden Kapitel werden die folgenden Fragen untersucht:

1. Wie wandelt sich der Anteil von Personen, die ein ehrenamtliches Engagement ausüben, über die Zeit? Zeigen sich für unterschiedliche Altersgruppen unterschiedliche Trends von 1996 bis 2014 ?

2. Engagieren sich verschiedene Bevölkerungsgruppen (nach Bildung, Geschlecht, Landesteil, Erwerbsstatus) unterschiedlich häufig und zeichnen sich diesbezüglich Veränderungen über die Zeit $a b$ ?

3. Wie unterscheiden sich Personen in seniorenspezifischen Gruppen von Personen mit einem allgemeinen ehrenamtlichen Engagement?

4. Verändern sich die Anteile der ehrenamtlich Engagierten, die innerhalb der letzten sechs Kalenderjahre ein Ehrenamt begonnen haben, über die Zeit und über die Altersgruppen?

len Engagementniveaus und der Veränderungen zwischen 1996 und 2014. Die Frage nach dem allgemeinen organisationsgebundenen ehrenamtlichen Engagement wurde an alle DEAS-Befragte gestellt und liegt somit für die Altersspanne 40 bis 85 Jahre vor. Ob Personen ein seniorenspezifisches Engagement ausüben, wurde jedoch erst ab einem Alter von 50 Jahren erfragt.

Engagementausübung. Um das organisationsgebundene Engagement zu erfassen, werden Personen zunächst gefragt, ob sie in einer Gruppe Mitglied sind, „die sich besonders an ältere Menschen im Ruhestand oder im Übergang in den Ruhestand" richtet (im Folgenden als Seniorengruppe bezeichnet) oder ob sie Mitglied in einer „ganz allgemein(en) Gruppe oder Organisation (sind), in der man Mitglied sein kann“ (im Folgenden als allgemeine Gruppen bezeichnet). Die Befragten können jeweils maximal fünf solcher Gruppen nennen, in denen sie Mitglied sind. Als 
ehrenamtliches Engagement wird es hier gewertet, wenn die Person weiterhin angibt „eine Funktion oder [...] ein Ehrenamt" in der Gruppe inne zu haben. Falls Personen sowohl eine Funktion in einer allgemeinen als auch einer Seniorengruppe ausüben, dann werden sie als engagiert in einer Seniorengruppe betrachtet. Um Aussagen über ehrenamtliches Engagement insgesamt treffen $\mathrm{zu}$ können, wird betrachtet, ob sich Personen entweder in einer seniorenspezifischen oder in einer allgemeinen Gruppe engagieren.

Engagementbeginn. Personen, die eine Funktion in einer Gruppe oder Organisation ausüben, wurden zudem gefragt, seit wann sie diese Funktion ausüben. Dies ermöglicht die Bildung eines Indikators, der angibt, ob die Person innerhalb der letzten sechs Kalenderjahre ein neues Ehrenamt aufgenommen hat. Dafür wird die Distanz zwischen Interviewjahr und Jahr des Beginns des Ehrenamtes berechnet. Übt eine Person mehrere Ehrenämter aus, zählt das zuletzt aufgenommene Ehrenamt. Im DEAS wird nicht gefragt, ob eine Person ein Engagement beendet hat. Deshalb können keine Ergebnisse hierzu berichtet werden. Jedoch haben Hank \& Erlinghagen (2010) gezeigt, dass die Beendigung eines Engagements durch ähnliche Prozesse bestimmt wird wie die Aufnahme eines Engagements.

Gruppierungsvariablen. Um Unterschiede im Bildungsniveau abzubilden, wird auf eine Unterscheidung zwischen Personen mit niedriger, mittlerer und höherer Bildung zurückgegriffen, die auf der ISCED-Skala beruht. Um das ehrenamtliche Engagement zwischen Personen unterschiedlichen Alters möglichst präzise vergleichen zu können, werden Sechs-Jahres-Altersgruppen genutzt ( 42 bis 47 Jahre, 48 bis 53 Jahre, 54 bis 59 Jahre, 60 bis 65 Jahre, 66 bis 71 Jahre, 72 bis 77 Jahre, 78 bis 83 Jahre). In Kombination mit dem Sechs-Jahres-Abstand zwischen den Befragungszeitpunkten hat dies weiterhin den Vorteil, dass sich die Altersgruppen und die Erhebungszeitpunkte nicht überschneiden. Da die Frage nach Seniorengruppen erst ab einem Alter von 50 Jahren gestellt wird und in der Altersgruppe ,84 und 85 Jahre' nur wenige Personen engagiert sind, werden die Ergebnisse teilweise nur für die 54- bis 83-jährigen Befragten dargestellt (vgl. Kapitel 2).

Erwerbsstatus. Auch der aktuelle Erwerbsstatus wurde im DEAS zu jedem Befragungszeitpunkt erfasst. Für die Analysen wird dieser in drei Gruppen unterteilt: Erwerbstätige (darunter fallen alle Personen, die in Teil- oder Vollzeit erwerbstätig sind, aber auch Personen, die unregelmäßig, geringfügig oder nebenerwerbstätig sind); Personen im Ruhestand, die Altersrente, Pension, sowie Frührente, -pension beziehen oder sich noch im Vorruhestand oder in der Freistellungsphase der Altersteilzeit befinden; und sonstige Nicht-Erwerbstätige (Arbeitslose sowie alle Personen ohne Erwerbseinkommen und ohne Rente wie Hausfrauen/-männer und aus anderen Gründen nicht Erwerbstätige).

Analysen. Die Abbildungen und Kennzahlen wurden mittels gewichteter Häufigkeiten berechnet. Die Analysen der Gruppenunterschiede beruhen auf Signifikanztests mittels logistischer Regression, wobei für die Stratifizierungsvariablen Altersgruppe, Geschlecht und Landesteil (Ost-/Westdeutschland) kontrolliert wurde. Das genaue Vorgehen ist in Kapitel 2 beschrieben.

\subsection{Veränderung von ehrenamtlichem Engagement zwischen den Jahren 1996 und 2014}

Zunächst wird auf die Veränderung von ehrenamtlichem Engagement über die Zeit eingegangen. Dabei werden neben dem allgemeinen Trend auch unterschiedliche Veränderungen nach Altersgruppen betrachtet.

\author{
Der Anteil von Personen, die in der zweiten \\ Lebenshälfte ein ehrenamtliches Engagement \\ ausüben, hat sich zwischen 1996 und 2014 \\ verdoppelt.
}


Ehrenamtliches Engagement hat zwischen 1996 und 2014, wie erwartet, an Bedeutung gewonnen. Im Jahr 1996 haben 11,7 Prozent der 40bis 85-Jährigen ein ehrenamtliches Engagement ausgeübt. Dieser Anteil unterschied sich nicht zu dem des Jahres 2002. Im Jahr 2008 lag er jedoch mit 18,2 Prozent deutlich höher. Der Anteil der Engagierten an den Personen dieser Altersgruppe erhöhte sich vom Jahr 2008 zum Jahr 2014 noch einmal auf 22,2 Prozent (Abbildung 5-1).

Im Folgenden wird betrachtet, ob diese $\mathrm{Zu}$ nahme über die Zeit für alle Altersgruppen in gleichem Maße erfolgt ist (Abbildung 5-1). Für alle Altersgruppen zeigt sich zwischen 1996 und 2014 eine Zunahme des Anteils von Engagierten. Der Anstieg ist jedoch für die höheren Altersgruppen besonders stark ausgeprägt: auch wenn bereits bei den 42 - bis 47 -Jährigen das ehrenamtliche Engagement zugenommen hat, steigt gerade in den Altersgruppen der 66- bis 71-Jährigen, der 72- bis 77-Jährigen und der 78- bis 83-Jährigen der Anteil der Engagierten über die Befragungszeitpunkte deutlich an. Das bedeutet, dass im Vergleich zum Jahr 1996 ein ehrenamtliches Engagement im Jahr 2014 ver- mehrt auch in höheren Altersgruppen ausgeübt wird. Dies lässt sich auch an den Veränderungen des Anteils der Engagierten über die Altersgruppen - getrennt nach Erhebungsjahren - ablesen. Im Jahr 1996 zeigte sich kein Unterschied zwischen den beiden jüngsten Altersgruppen der 42- bis 47 -Jährigen und der 48 - bis 53-Jährigen, jedoch wiesen die älteren Altersgruppen deutlich geringere Anteile an ehrenamtlich Engagierten auf. Im Jahr 2014 unterscheiden sich die Altersgruppen zwischen den 42 - bis 65 -Jährigen nicht. Die 66- bis 71-Jährigen üben sogar häufiger als die jüngeren Altersgruppen ein Engagement aus, die 72- bis 77-Jährigen weisen wieder das Niveau der 42- bis 65-Jährigen auf. Erst in der ältesten Altersgruppe der 78- bis 83-Jährigen ist der Anteil der Engagierten deutlich geringer. Damit zeigt sich, dass im Jahr 2014 ein ehrenamtliches Engagement bis ins Alter von 70 Jahren etwa gleich häufig ausgeübt wird. Die relativ stabilen Anteile von Personen mit ehrenamtlichem Engagement von den mittleren $\mathrm{zu}$ den höheren Altersgruppen sind somit ein relativ junges Phänomen.

Abbildung 5-1: Anteile der ehrenamtlich Engagierten, gesamt und nach Alter, in den Jahren 1996, 2002, 2008, 2014 (in Prozent)

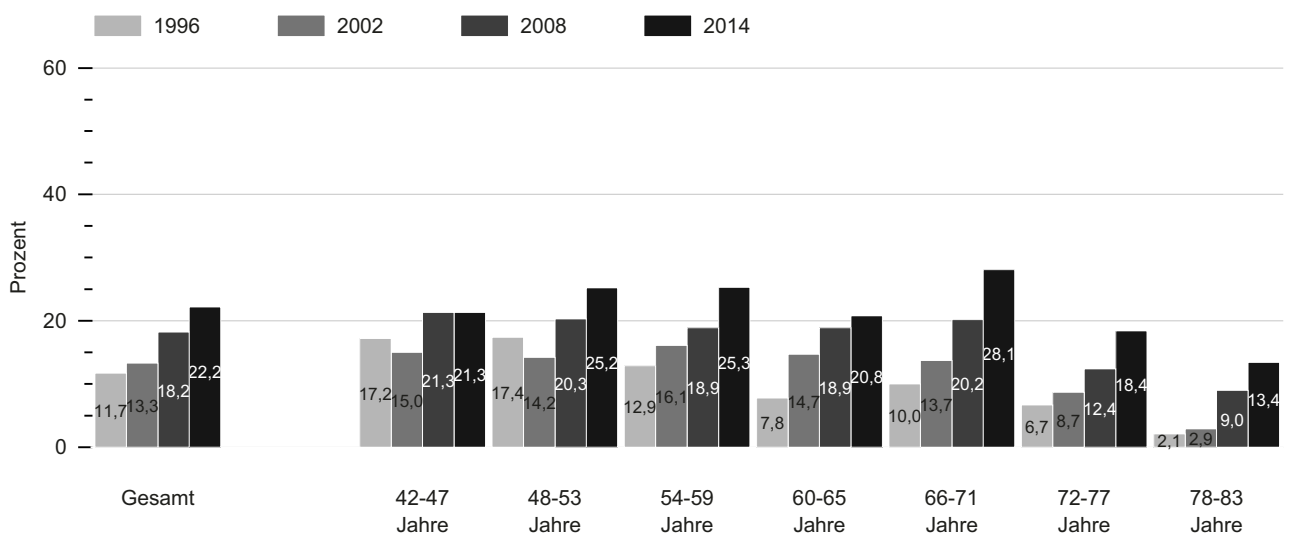

Quelle: DEAS 1996 ( $n=4.808), 2002(n=3.039), 2008$ ( $n=6.194), 2014(n=5.993)$ gewichtet, gerundete Angaben; $(p<, 05)$. Nur zwischen 1996 und 2002 kein signifikanter Unterschied über alle Altersgruppen insgesamt. Signifikante Unterschiede zwischen folgenden Altersgruppen: 42 bis 47 Jahre bzw. 60 bis 65 Jahre zu 66 bis 71 Jahre und 48 bis 59 Jahre zu 72 bis 83 Jahre sowie 42 bis 77 Jahre zu 78 bis 83 Jahre. Signifikante Interaktion zwischen Erhebungsjahr und Alter. 


\subsection{Wandel vom Anteil von ehrenamtlichem Engagement nach Bevölkerungsgruppen zwischen 1996 und 2014}

Im Folgenden wird betrachtet, ob sich die Anteile der Personen, die sich ehrenamtlich engagieren, nach Bevölkerungsgruppen unterscheiden. Dabei werden vor allem Unterschiede nach Geschlecht, Bildungsgruppen, Landesteil und Erwerbsstatus betrachtet.

\section{Im Jahr 2014 zeigen sich deutliche Unterschiede im ehrenamtlichen Engagement nach Geschlecht, Bildung, Landesteil und Erwerbsstatus.}

Für das Jahr 2014 zeigen sich deutliche Unterschiede nach Geschlecht (Abbildung 5-2). So engagieren sich 25,6 Prozent der Männer und nur 19,0 Prozent der Frauen in einem organisationsgebundenen Ehrenamt. Von den Personen mit niedrigerem Bildungsniveau üben 8,0 Prozent ein ehrenamtliches Engagement aus, während von den Personen mit hohem Bildungsniveau 28,8 Prozent ein Ehrenamt ausüben. Damit ist der Anteil der Engagierten mit hoher Bildung um ein 3,6-faches höher als der Anteil von Personen mit niedrigerer Bildung. Schließlich bestehen Unterschiede nach Landesteilen: 23,9 Prozent der Personen im Alter von 40 bis 85 Jahren, die in Westdeutschland leben, üben ein ehrenamtliches Engagement aus. In Ostdeutschland sind es 14,3 Prozent.

Weiterhin sind im Jahr 2014 24,5 Prozent der Erwerbstätigen, aber nur 17,2 Prozent der Nicht-Erwerbstätigen im Alter von 40 bis 85 Jahren, ehrenamtlich engagiert (Abbildung 5-2). Die Erwerbstätigen liegen auf gleichem Niveau wie Personen im Ruhestand, sodass auch die Personen im Ruhestand häufiger ehrenamtlich engagiert sind als die nicht-erwerbstätigen Personen. An den gezeigten Niveauunterschieden im Anteil an ehrenamtlich Engagierten zeigt sich, dass Personen mit mehr Ressourcen und besseren Gelegenheitsstrukturen häufiger einem ehrenamtlichen Engagement nachgehen.

Abbildung 5-2: Anteile der ehrenamtlich Engagierten, nach Geschlecht, Bildung, Landesteil und Erwerbsstatus, im Jahr 2014 (in Prozent)

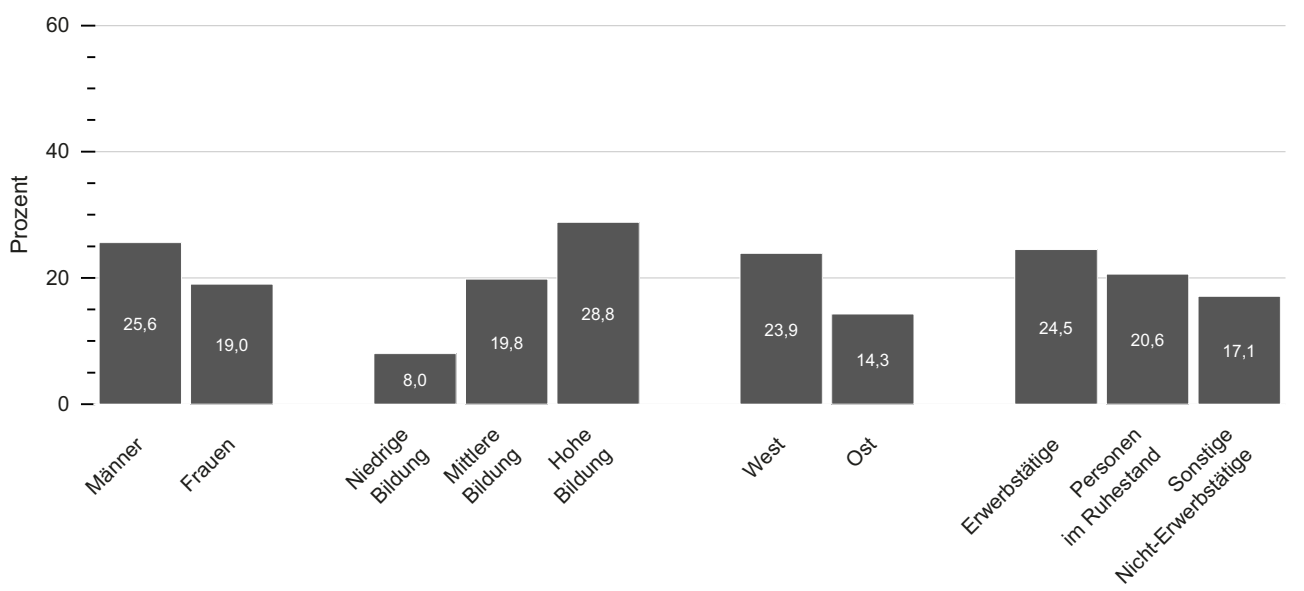

Quelle: DEAS 2014 ( $n=5.993)$ gewichtet, gerundete Angaben. $(p<, 05)$.

Signifikante Unterschiede nach Geschlecht, Bildung und Landesteil. Erwerbstätige und Personen im Ruhestand unterscheiden sich nicht bedeutsam, jedoch weisen die Sonstigen Nicht-Erwerbstätigen einen geringeren Anteil auf. 
Die Unterschiede im ehrenamtlichen Engagement zwischen Männer und Frauen haben zwischen den Jahren 1996 und 2014 abgenommen, jedoch sind die Unterschiede nach Bildungshintergrund und Landesteil gleichgeblieben.

Um zu analysieren, ob sich die Teilhabe am ehrenamtlichen Engagement zwischen 1996 und 2014 für einige Gruppen besonders stark verändert hat, können Veränderungen über die Zeit auch getrennt für verschiedene Bevölkerungsgruppen betrachtet werden. Es zeigt sich, dass sowohl Männer als auch Frauen im Jahr 2014 deutlich häufiger ein ehrenamtliches Engagement als noch im Jahr 1996 ausüben. Gleichzeitig verringern sich die Unterschiede zwischen Männern und Frauen, denn betrug der Abstand im Jahr 1996 noch 9,1 Prozentpunkte lag der Unterschied im Jahr 2014 nur noch bei 6,6 Prozentpunkten. Diese Abnahme lässt sich vor allem darauf zurückführen, dass der Anteil der engagierten Frauen (1996: 7,6 Prozent; 2014: 19,0 Prozent) von einem niedrigeren Niveau aus stärker gewachsen ist als der der Männer (1996: 16,6 Prozent; 2014: 25,6 Prozent).

Zwischen Bildungsgruppen und Landesteilen haben sich die Unterschiede im ehrenamtlichen Engagement zwischen 1996 und 2014 nicht verändert (ohne Abbildung). Alle Gruppen zeigen eine ähnlich starke Zunahme.

Der Anteil der Engagierten wächst zwischen 1996 und 2014 ebenfalls über alle Erwerbsstatusgruppen. Während jedoch der Anteil der Engagierten unter den Nicht-Erwerbstätigen nur von 10,5 Prozent im Jahr 1996 auf 17,1 Prozent im Jahr 2014 gestiegen ist, verbuchen Erwerbstätige (1996: 15,8 Prozent; 2014: 24,5 Prozent) und insbesondere Personen im Ruhestand (1996: 7,7 Prozent; 2014: 20,5 Prozent) stärkere Zuwächse (ohne Abbildung). Damit sinkt der Unterschied im Anteil der Engagierten zwischen den Erwerbstätigen und den Personen im Ruhestand deutlich von 8,0 Prozentpunkten im Jahr 1996 auf 4,0 Prozentpunkte im Jahr 2014, wobei der Unterschied zwischen den Erwerbstätigen und den sonstigen Nicht-Erwerbstätigen im gleichen Zeitraum von 5,3 auf 7,4 Prozentpunkte zunimmt.

\subsection{Unterschiede von seniorenspezifischem Engagement zu ehrenamtlichem Engagement insgesamt im Jahr 2014}

Im Folgenden soll nun betrachtet werden, ob sich diejenigen, die sich in einer seniorenspezifischen Gruppe engagieren von den bis hierher berichteten Engagierten insgesamt unterscheiden. Da Personen nur dann gefragt wurden, ob sie sich in einer Gruppe engagieren, die sich speziell an Seniorinnen und Senioren richtet, wenn sie älter als 50 Jahre waren, beziehen sich die kommenden Ergebnisse auf diese Altersgruppe. Die Engagementquote insgesamt beträgt im Jahr 2014 auch in der Altersgruppe der 50 bis 85-Jährigen 22,2 Prozent.

In einer Seniorengruppe engagieren sich im Jahr 2014 5,6 Prozent der Personen im Alter von 50 bis 85 Jahren. Die Übernahme einer Funktion in einer Seniorengruppe ist ungleich über die Altersgruppen verteilt (Abbildung 5-3). In den Altersgruppen der 54- bis 59- und 60- bis 65-Jährigen sind ehrenamtliche Engagements in Seniorengruppen gleichhäufig. Davon unterscheiden sich die Altersgruppen ab 66 Jahre. In diesen gehen Personen häufiger einem seniorenspezifischen Engagement nach.

Im Jahr 2014 ist jede vierte ehrenamtlich engagierte Person im Alter von $\mathbf{5 0}$ bis 85 Jahren in einer Seniorengruppe aktiv.

Von den Engagierten insgesamt in dieser Altersgruppe übt mit 25,4 Prozent circa jede vierte Person ein seniorenspezifisches Engagement aus (nicht abgebildet). Es zeigt sich, dass der Anteil der Engagierten in Seniorengruppen 
an allen Engagierten mit höherem Alter höher ist. Bei den Personen zwischen 54 und 59 Jahren sind 15,8 Prozent der Engagierten in einer Seniorengruppe aktiv, wohingegen dies in der Altersgruppe der 78- bis 83-Jährigen sogar 51,4 Prozent der Engagierten sind.

Weiterhin zeigt sich, dass sich im Jahr 2014 Männer und Frauen gleichhäufig in seniorenspezifischen Gruppe engagieren (Abbildung 5-3). Dies steht im Gegensatz zum Engagement insgesamt, bei dem sich Männer zu einem höheren Anteil engagieren als Frauen. Unterschiede im seniorenspezifischen Engagement zeigen sich jedoch nach Bildungsniveau und Landesteil. Ebenso wie beim Engagement insgesamt sind Personen mit hohem Bildungsniveau zu einem größeren Anteil in Seniorengruppen engagiert als Personen mit mittlerem oder niedrigerem Bildungsniveau. Ebenfalls üben mehr Personen in Westdeutschland ein seniorenspezifisches Engagement aus als in Ostdeutschland. Diese Unterschiede bestehen über alle Altersgruppen. Somit folgt seniorenspezifisches Engagement in Bezug auf Bildungsniveau und Landesteil den gleichen Verteilungsmustern wie das ehrenamtliche Engagement insgesamt, jedoch trägt es zwischen Männern und Frauen zu einer Reduktion der Unterschiede im Engagement insgesamt bei.

Der Unterschied im seniorenspezifischen Engagement zwischen den Altersgruppen spiegelt sich auch im Erwerbsstatus wider: Personen im Ruhestand engagieren sich im Jahr
2014 mit einem Anteil von 7,9 Prozent deutlich häufiger in Seniorengruppen als Erwerbstätige (2,9 Prozent) und sonstige Nicht-Erwerbstätige (4,5 Prozent). Letztere unterscheiden sich statistisch nicht voneinander (nicht abgebildet).

\section{Engagement in Seniorengruppen ist zwischen den Jahren 1996 und 2014 - analog zum Gesamttrend - häufiger geworden.}

Der Anteil der Engagierten in Seniorengruppen ist von 2,1 Prozent im Jahr 1996 auf 5,6 Prozent im Jahr 2014 häufiger geworden. Dieser Trend verläuft proportional zum allgemeinen Engagement. Somit werden Seniorengruppen zwar über die Erhebungsjahre immer häufiger ausgeübt, jedoch sind sie innerhalb des ehrenamtlichen Engagements insgesamt zwischen 1996 und 2014 nicht bedeutsamer geworden. Dies zeigt sich auch für die Altersgruppen nach dem Ruhestandsübergang, in denen der Anstieg von seniorenspezifischem Engagement sowie im Engagement insgesamt besonders stark war. Ebenfalls zeigen sich nach Geschlecht, Bildung, Landesteil und Erwerbsstatus keine Verschiebungen im Anteil des seniorenspezifischen Engagements am ehrenamtlichen Engagement insgesamt. Auch wenn sich alle Bevölkerungsgruppen häufiger in einer seniorenspezifischen Gruppe engagieren, hat sich der Anteil der Seniorengruppen am Engagement insgesamt zwischen 1996 und 2014 nicht verändert. 
Abbildung 5-3: Anteile der ehrenamtlich Engagierten in Seniorengruppen, nach Alter, Geschlecht, Bildung und Landesteil, im Jahr 2014 (in Prozent)

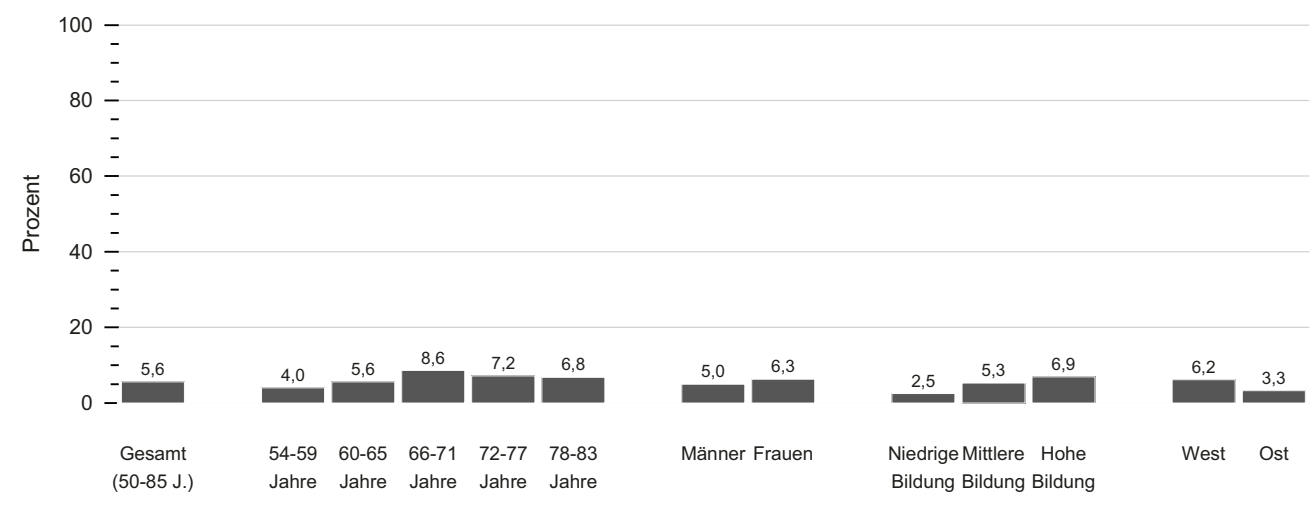

Quelle: DEAS 2014 ( $n=5.993)$ gewichtet, gerundete Angaben; $(p<, 05)$.

Signifikante Unterschiede nur zwischen den Altersgruppen 54 bis 59 Jahre bzw. 60 bis 65 Jahre zu 66 bis 71 Jahre und 54 bis 59 Jahre zu 72 bis 77 Jahre bzw. 78 bis 83 Jahre. Kein Geschlechtsunterschied. Signifikante Unterschiede nach Bildung und Landesteil.

\subsection{Veränderung von Neuaufnahmen von ehrenamtlichem Engagement zwischen den Jahren 1996 und 2014}

Im Folgenden wird betrachtet, wie sich die Neuaufnahme des organisationsgebundenen ehrenamtlichen Engagements über die Zeit verändert hat. Dafür wird betrachtet, wer in den letzten sechs Kalenderjahren ein ehrenamtliches Engagement begonnen hat, welches zum Befragungszeitpunkt noch ausgeübt wird. Die Befunde werden in einem zweiten Schritt ins Verhältnis zum insgesamt ausgeübten Engagement gesetzt, sodass auch Aussagen über den Anteil des neuausgeübten am gesamten ehrenamtlichen Engagement und damit über die Stabilität des Engagements möglich werden.

\section{Ein Drittel der im Jahr 2014 ehrenamtlich Engagierten hat in den letzten sechs Jahren ein neues Engagement aufgenommen und zwei Drittel üben ihr Engagement schon länger als sechs Jahre aus.}

Für das Jahr 2014 zeigt sich, dass 7,1 Prozent der Personen in der zweiten Lebenshälfte seit 2009 (mindestens) ein ehrenamtliches Engagement aufgenommen haben, welches sie noch immer ausüben. Das heißt, dass von den 22,2 Prozent der im Jahr 2014 ehrenamtlich engagierten Personen im Alter von 40 bis 85 Jahren ein Drittel seit 2009 ein (zusätzliches) Engagement begonnen hat. Im Umkehrschluss bedeutet das, dass mindestens zwei Drittel der im Jahr 2014 Engagierten ihr Engagement bereits länger als sechs Jahre ausüben. Der Anteil von Personen mit einem neuen Engagement am Engagement insgesamt könnte sogar noch geringer sein, da hier nur das zuletzt begonnene Engagement betrachtet wird. Personen mit einem neu begonnenen Engagement können jedoch bereits ein anderes Engagement ausüben. Im Jahr 2014 traf dies auf circa ein Drittel der seit dem Jahr 2009 Neuengagierten zu.

Der Anteil der Männer, die seit dem Jahr 2009 ein Ehrenamt aufgenommen haben, das 
sie 2014 noch ausüben, ist ebenso hoch wie der Anteil von Frauen (Abbildung 5-4). Von den niedriger Gebildeten üben im Jahr 2014 nur 1,5 Prozent ein Engagement aus, welches sie seit 2009 begonnen haben. Für Personen mit mittlerem Bildungsniveau liegt der Anteil deutlich höher bei 5,6 Prozent und bei Hochgebildeten sogar bei 10,5 Prozent. Auch zwischen den Landesteilen zeigt sich ein bedeutsamer Unterschied: Von den Personen in Westdeutschland in der zweiten Lebenshälfte üben noch 7,7 Prozent eines der organisationsgebundenen ehrenamtlichen Engagements aus, die sie in den letzten sechs Kalenderjahren begonnen haben, wohingegen dieser Anteil unter den Personen aus Ostdeutschland nur 4,1 Prozent beträgt. Der Anteil der Neuengagierten an den Engagierten, das heißt ob Personengruppen anteilig häufiger einem neuen Engagement aus den letzten sechs Jahren nachgehen als andere Personen, unterscheidet sich - analog zum Gesamttrend - nicht nach Geschlecht, Bildung und Landesteil.

\section{Personen höheren Alters nehmen zu geringeren Anteilen ein Engagement auf als jüngere Personen, scheinen dies jedoch dafür länger auszuüben.}

Im Jahr 2014 liegt der Anteil der 42- bis 70-Jährigen, die in den letzten sechs Kalenderjahren ein organisationsgebundenes Engagement begonnen haben bei über 7 Prozent. Für die 72bis 77-Jährigen liegen die Neuaufnahmen bei 3,4 Prozent und für die 78- bis 83-Jährigen bei 1,3 Prozent. Da die neuaufgenommenen Engagements mit höherem Alter geringer sind als die ehrenamtlichen Engagements insgesamt, ist der Anteil der Neuengagierten an allen Engagierten im höheren Alter geringer als im mittleren Alter: Unter den 42- bis 47-Jährigen, die im Jahr 2014 mindestens ein ehrenamtliches Engagement ausüben, haben 51,5 Prozent mindestens ein Engagement erst in den letzten sechs Jahren begonnen (ohne Abbildung). Bei den 72bis 77-Jährigen (und 78- bis 83 -Jährigen) beträgt dieser Anteil nur noch 18,6 Prozent (und 9,4 Prozent). Das bedeutet, dass ältere Personen stabiler einem einmal bestehenden Engagement nachgehen als jüngere Personen.

\section{Zwischen 1996 und 2014 hat sich der Anteil der Neuengagierten an allen Engagierten nicht bedeutsam verändert.}

Der Anteil der Neuengagierten an allen Personen in der zweiten Lebenshälfte kann auch über die Zeit betrachtet werden. Im Vergleich zu 2014, in dem 7,1 Prozent der Personen in den letzten sechs Kalenderjahren ein neues Engagement aufgenommen, haben im Jahr 1996 nur 3,6 Prozent der Personen ein Engagement ausgeübt, welches sie frühestens 1991 begonnen hatten. Für eine Veränderung der Stabilität des gesellschaftlichen Engagements zwischen 1996 und 2014 gibt es jedoch keinen Hinweis, da über den Zeitraum der Anteil der Neuengagierten an den Gesamtengagierten bei circa 31 Prozent konstant geblieben ist. Die Ergebnisse deuten darauf hin, dass mit dem Anstieg des ehrenamtlichen Engagements zwischen 1996 und 2014 es nicht primär zu einer Ausdehnung des eher kurzfristigen oder des eher langfristigen Engagements gekommen ist, sondern das Verhältnis (auch über unterschiedliche gesellschaftliche Gruppen) relativ stabil geblieben ist. 
Abbildung 5-4: Anteile der Personen, die in den letzten sechs Kalenderjahren ein Engagement aufgenommen haben, gesamt, nach Alter, Geschlecht, Bildung und Landesteil, im Jahr 2014 (in Prozent)

\section{a) Gesamt und nach Alter}

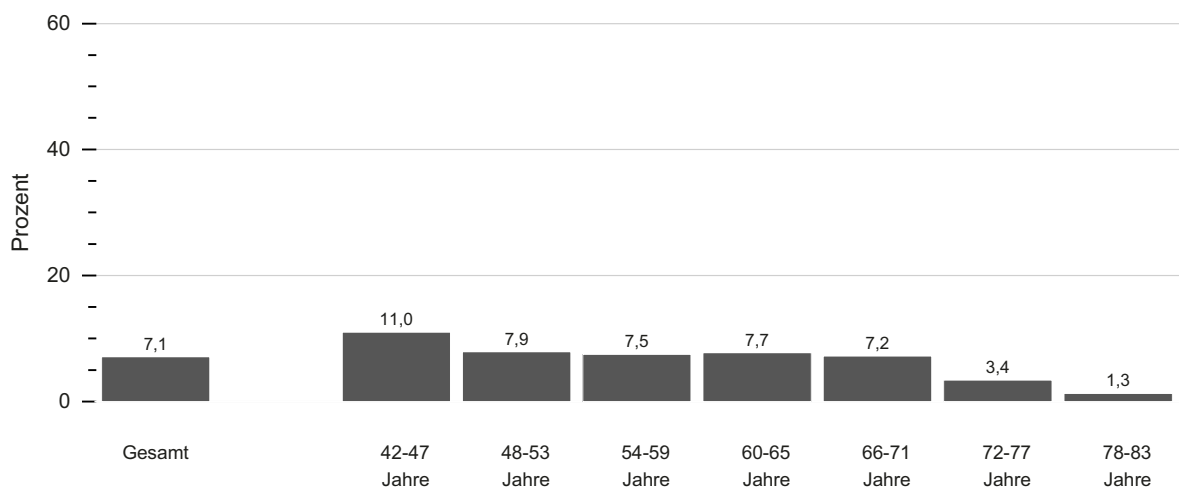

b) Nach Geschlecht, Bildung und Landesteil

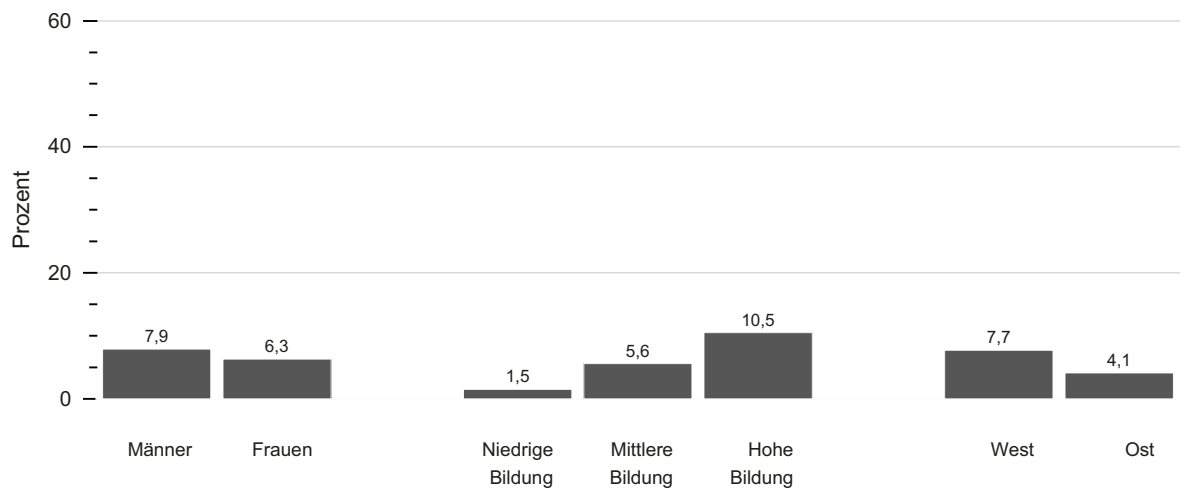

Quelle: DEAS 2014 ( $n=5.993$ ) gewichtet, gerundete Angaben; $(p<, 05)$.

a) Signifikante Altersunterschiede für alle Gruppen außer zwischen den Gruppen der 48- bis 71-Jährigen. b) Kein Geschlechtsunterschied. Signifikante Unterschiede nach Bildung und Landesteil.

\subsection{Diskussion und Implikationen}

Zwischen den Jahren 1996 und 2014 haben die Anteile derer, die in der zweiten Lebenshälfte ein ehrenamtliches Engagement ausüben, deutlich zugenommen. $\mathrm{Da}$ in diesem Kapitel das organisationsgebundene Engagement betrachtet wurde und gerade nicht-organisationsgebundenes Engagement in den letzten Jahren stark gestiegen ist (vgl. Sachße 2011), können die beschriebenen Trends sogar als konservativ betrachtet werden.

Der Anstieg der Engagementquote zwischen den Jahren 1996 und 2014 kann durch drei Trends charakterisiert werden. Erstens konnte eine allgemeine Erhöhung des Anteils der Enga- 
gierten an der Bevölkerung im Alter von 40 bis 85 Jahre gezeigt werden, der über alle betrachteten Alters- und Bildungsgruppen sowohl für West- und Ostdeutschland als auch für Männer und Frauen zutrifft. Zweitens steigt speziell der Anteil der Engagierten in höheren Altersgruppen. Davon profitieren besonders Personen im Ruhestand und das seniorenspezifische Engagement. Und drittens ist zwischen 1996 und 2014 der Anteil von älteren Erwerbstätigen gestiegen (vgl. Kapitel 3), die überproportional häufig zusätzlich ein ehrenamtliches Engagement ausüben. Dafür, dass sich die Stabilität von ehrenamtlichen Engagement geändert hat, konnten keine Hinweise gefunden werden.

Diese drei Trends lassen sich unterschiedlichen Zugangsstrukturen zu ehrenamtlichem Engagement zuordnen. Der allgemeine Anstieg in Form eines ,Fahrstuhl-Effektes' lässt sich auf gesamtgesellschaftliche Veränderungen zurückführen, da alle Subgruppen der Bevölkerung in einem ähnlichen Maße davon betroffen sind. Es kann davon ausgegangen werden, dass dies durch eine Normenverschiebung zu einer breiteren Akzeptanz von ehrenamtlichem Engagement zustande gekommen ist, die auch ein Resultat gezielter , aktivierungspolitischer' Anstrengungen in den letzten Jahrzehnten sein kann. Der Anstieg ehrenamtlichen Engagements gerade im höheren Lebensalter wird vermutlich besonders durch einen Gewinn an Gesundheitsressourcen über die letzten Jahre vorangetrieben. In Kapitel 8 wird gezeigt, dass Personen im höheren Lebensalter im Jahr 2014 seltener als 1996 gesundheitliche Einschränkungen aufweisen. Das verlängerte Aufrechterhalten von wichtigen Ressourcen für ein ehrenamtliches Engagement könnte bewirkt haben, dass im Jahr 2014 der Anteil des ausgeübten, aber auch des neu aufgenommenen Engagements erst in den Altersgruppen 70 Jahre und älter sinkt. Davon profitieren besonders auch Seniorengruppen, deren Bedeutung im selben Zeitraum absolut (jedoch nicht überproportional) gewachsen ist. Ebenfalls wirken aktuelle Trends des Arbeitsmarktes positiv auf die Ausübung von ehrenamtlichem Engagement. Erwerbstätige haben bereits 1996 überproportional häufig ein ehrenamtliches Engagement ausgeübt. Der Anteil der
Engagierten ist unter den Erwerbstätigen jedoch noch einmal deutlich gestiegen. Dies wirkt sich zweifach auf die allgemeine Engagementquote aus, da nicht nur der Anteil der Engagierten in dieser Gruppe steigt, sondern auch mehr ältere Personen sich noch in Erwerbstätigkeit befinden (vgl. Kapitel 3).

Besonders interessant sind die Parallelen in der Entwicklung der Erwerbsbeteiligung, wie in Kapitel 3 dargestellt, und der Entwicklung des ehrenamtlichen Engagements zwischen den Jahren 1996 und 2014. Männer sind nicht nur häufiger ehrenamtlich engagiert, sondern gehen auch häufiger einer Erwerbstätigkeit nach als Frauen. Diese Geschlechtsunterschiede haben sowohl für Engagement als auch für Erwerbstätigkeit seit dem Jahr 1996 abgenommen. Die Unterschiede nach Bildungsniveau- und Landesteil, die bereits 1996 sowohl in der Erwerbsals auch in der Engagementbeteiligung bestanden, haben sich jedoch bis ins Jahr 2014 nicht verändert. Die Parallelen im Wandel über die Zeit lassen sich durch zwei Argumentationslinien erklären: Einerseits bildet die Erwerbstätigkeit eine Gelegenheitsstruktur für ehrenamtliches Engagement. Daraus könnte folgen, dass ein Anstieg von Erwerbstätigen auch eine steigende Engagementquote nach sich zieht. Andererseits können beide Teilhabeformen aber auch die gleichen Voraussetzungen haben, sodass eine Suche nach gemeinsamen Mechanismen der Partizipation, wie zum Beispiel Gesundheit, Ressourcen, Fähigkeiten und Interessen, sowohl die Einbindung in das eine als auch in das andere vorhersagen könnten.

Da ehrenamtliches Engagement in der richtigen Häufigkeit positive Auswirkungen auf die Lebensqualität haben kann (vgl. Windsor, Anstey et al. 2008), wirkt sich eine systematische Nichtteilhabe von Gruppen, die bereits sozial benachteiligt sind, vermutlich weiter verstärkend auf bestehende soziale Unterschiede aus. So werden beispielsweise Personen, die nicht erwerbstätig sind, sowohl mit den nachteiligen Folgen der Erwerbs- als auch der Engagementlosigkeit konfrontiert. Strukturelle Unterschiede (zum Beispiel in der Infrastruktur oder im Arbeitsmarkt) zwischen den Landesteilen, die ebenfalls die Gelegenheitsstrukturen für ehren- 
amtliches Engagement bilden, können die bestehenden Unterschiede zwischen Personen in Ost- und Westdeutschland weiter vergrößern. Ähnliche Unterschiede im ehrenamtlichen Engagement zeigen sich auch zwischen Männern und Frauen, jedoch reduzieren sie sich zwischen den Jahren 1996 und 2014.

Bestehende Unterschiede nach Gruppen sollten weiter abgebaut werden, da daraus systematische Nachteile in den Lebenschancen entstehen können. Auch aus einer gesellschaftlichen Perspektive sind ungenutzte Potenziale nachteilig. Eine Angleichung der Lebensbedingungen und damit der Gelegenheitsstrukturen zwischen den beiden deutschen Landesteilen genauso wie ein Abbau von Bildungs- und Geschlechtsunterschieden könnten daher primäre Ziele kommender Engagementpolitik sein. Die Förderung von seniorenspezifischem Engagement kann dabei nur ein Aspekt sein, da dieses zwar leicht die Engagementunterschiede zwischen den Geschlechtern reduziert, ansonsten jedoch durch die gleichen Merkmale wie Engagement insgesamt vorhergesagt wird. Das bedeutet, dass eine Förderung von seniorenspezifischem Engagement Unterschiede zwischen den Landesteilen und zwischen Bildungsgruppen nicht verringern würde.

Ein Abbau von Ungleichheiten auf dem Arbeitsmarkt könnte sich in doppelter Weise positiv auswirken, da die Gelegenheitsstrukturen, die die Erwerbsarbeit bietet, sich auch positiv auf ehrenamtliches Engagement auszuwirken scheinen. Da die Gruppenunterschiede im ehrenamtlichen Engagement bereits zu Beginn der zweiten Lebenshälfte bestehen und über diese konstant bleiben, sollte an einem früheren Zeitpunkt im Lebenslauf angesetzt werden (Warner, Wolff et al. 2014). Wird das Entstehen von sozialen Unterschieden bereits zu einem biografisch früheren Zeitpunkt reduziert, könnte von den positiven Auswirkungen des Engagements ein größerer Teil der Bevölkerung langfristig profitieren.

\section{Literatur}

Alscher, M., Dathe, D., Eckhard, P., \& Speth, R. (2009). Bericht zur Lage und zu den Perspektiven des bürgerschaftlichen Engagements in Deutschland. Berlin: Wissenschaftszentrum Berlin für Sozialforschung.

Dyk, S. van (2009). Das Alter: adressiert, aktiviert, diskriminiert. Berliner Journal für Soziologie, 19(4), 601-625. doi: 10.1007/s11609-009-0114-z.

Erlinghagen, M. (2010). Volunteering after retirement: Evidence from German panel data. European Societies, 12(5), 603-625.

Europäische Union (2012). Europäisches Jahr des aktiven Alterns und der Solidarität zwischen den Generationen. Online: http://ec.europa.eu/social/main.jsp? cat $\mid d=1002$ \&langld=de (zuletzt abgerufen 15.07.2016).

Everard, K. M., Lach, H. W., Fisher, E. B., \& Baum, M. C. (2000). Relationship of Activity and Social Support to the Functional Health of Older Adults. The Journals of Gerontology Series B: Psychological Sciences and Social Sciences, 55(4), S208-S212. doi: 10.1093/ geronb/55.4.S208.

Fünfter Altenbericht (2006). Potenziale des Alters in Wirtschaft und Gesellschaft. Der Beitrag älterer Menschen zum Zusammenhalt der Generationen. Fünfter
Bericht zur Lage der älteren Generation in der Bundesrepublik Deutschland. Berlin: Bundesministerium für Familie, Senioren, Frauen und Jugend.

Gesthuizen, M., \& Scheepers, P. (2012). Educational differences in volunteering in cross-national perspective: Individual and contextual explanations. Nonprofit and Voluntary Sector Quarterly, 41(1), 58-81.

Hagen, C., \& Vogel, C. (2012). Freiwilliges und generationenübergreifendes Engagement von Frauen und Männern - Analysepotenziale und Weiterentwicklung des Deutschen Freiwilligensurveys. Informationsdienst Altersfragen, 39(3), 3-9.

Hank, K., \& Erlinghagen, M. (2010). Dynamics of Volunteering in Older Europeans. The Gerontologist, 50(2), 170-178. doi: 10.1093/geront/gnp122.

Hank, K., \& Stuck, S. (2008). Volunteer Work, Informal Help, and Care among the 50+ in Europe: Further Evidence for 'Linked' Productive Activities at Older Ages. Social Science Research, 37, 1280-1291.

Hartnuß, B., Olk, T., \& Klein, A. (2011). Engagementpolitik. In: T. Olk \& B. Hartnuß (Hrsg.) Handbuch Bürgerschaftliches Engagement (S. 761-776). Weinheim: Beltz Juventa. 
Lancee, B., \& Radl, J. (2014). Volunteering over the life course. Social Forces, 93(2), 833-862.

Laubach, W., Schumacher, J., Mundt, A., \& Brähler, E. (2000). Sozialschicht, Lebenszufriedenheit und Gesundheitseinschätzung. Ergebnisse einer repräsentativen Untersuchung der deutschen Bevölkerung. Sozialund Präventivmedizin/Social and Preventive Medicine, 45(1), 2-12. doi: 10.1007/bf01358994.

Mutchler, J. E., Burr, J. A., \& Caro, F. G. (2003). From paid worker to volunteer: Leaving the paid workforce and volunteering in later life. Social Forces, 81(4), 12671293.

Naumann, D., \& Romeu Gordo, L. (2010). Gesellschaftliche Partizipation: Erwerbstätigkeit, Ehrenamt und Bildung. In: A. Motel-Klingebiel, S. Wurm \& C. TeschRömer (Hrsg.) Altern im Wandel. Befunde des Deutschen Alterssurveys (DEAS) (S. 118-141). Stuttgart: Kohlhammer.

Quaranta, M. (2015). Life Course, Gender, and Participation in Voluntary Organizations in Italy. Voluntas: International Journal of Voluntary and Nonprofit Organizations, online first, 27(2), 874-899.

Rosenbladt, B. von (2000). Volunteering in Germany: Results of the 1999 representative survey on volunteering and civic engagement.: Bundesministerium für Familie, Senioren, Frauen und Jugend \& Robert Bosch Stiftung.

Ross, C. E., \& Wu, C.-I. (1995). The Links Between Education and Health. American Sociological Review, 60(5), 719-745. doi: 10.2307/2096319.

Sachße, C. (2011). Traditionslinien bürgerschaftlichen Engagements in Deutschland. In: T. Olk \& B. Hartnuß (Hrsg.) Handbuch Bürgerschaftliches Engagement. Weinheim: Beltz Juventa.
Scherger, S., Nazroo, J., \& Higgs, P. (2011). Leisure activities and retirement: do structures of inequality change in old age? Ageing \& Society, 31, 146-172. doi: $10.1017 /$ S0144686X10000577.

Simonson, J., Hagen, C., Vogel, C., \& Motel-Klingebiel, A. (2013). Ungleichheit sozialer Teilhabe im Alter. Zeitschrift für Gerontologie und Geriatrie, 46(5), 410-416.

Warner, L. M., Wolff, J. K., Ziegelmann, J. P., \& Wurm, S. (2014). A randomized controlled trial to promote volunteering in older adults. Psychology and aging, 29(4), 757.

Willigen, M. van (2000). Differential Benefits of Volunteering Across the Life Course. The Journals of Gerontology Series B: Psychological Sciences and Social Sciences, 55(5), S308-S318. doi: 10.1093/geronb/55.5.S308.

Wilson, J. (2000). Volunteering. Annual Review of Sociology, 26, 215-240.

Wilson, J., \& Musick, M. (1997). Who cares? Toward an integrated theory of volunteer work. American Sociological Review, 62(5), 694-713.

Windsor, T. D., Anstey, K. J., \& Rodgers, B. (2008). Volunteering and Psychological Well-Being Among YoungOld Adults: How Much Is Too Much? The Gerontologist, 48, 59-70.

World Health Organization (2002). Active Ageing: A Policy Framework. Genf: World Health Organization.

Zimmer, A., \& Rauschenbach, T. (2011). Bürgerschaftliches Engagement unter Druck? Eine Einleitung. In: A. Zimmer \& T. Rauschenbach (Hrsg.) Bürgerschaftliches Engagement unter Druck? Analysen und Befunde aus den Bereichen Soziales, Kultur und Sport (S. 11-28). Opladen: Budrich.

Open Access Dieses Kapitel wird unter der Creative Commons Namensnennung 2.5 International Lizenz (http://creativecommons.org/licenses/by/2.5/deed.de) veröffentlicht, welche die Nutzung, Vervielfältigung, Bearbeitung, Verbreitung und Wiedergabe in jeglichem Medium und Format erlaubt, sofern Sie den/die ursprünglichen Autor(en) und die Quelle ordnungsgemäß nennen, einen Link zur Creative Commons Lizenz beifügen und angeben, ob Änderungen vorgenommen wurden.

Die in diesem Kapitel enthaltenen Bilder und sonstiges Drittmaterial unterliegen ebenfalls der genannten Creative Commons Lizenz, sofern sich aus der Abbildungslegende nichts anderes ergibt. Sofern das betreffende Material nicht unter der genannten Creative Commons Lizenz steht und die betreffende Handlung nicht nach gesetzlichen Vorschriften erlaubt ist, ist für die oben aufgeführten Weiterverwendungen des Materials die Einwilligung des jeweiligen Rechteinhabers einzuholen. 